\title{
Osteogenic markers are reduced in bone-marrow mesenchymal cells and femoral bone of young spontaneously hypertensive rats
}

\author{
Thamine Landim de Barros ${ }^{\mathrm{a}, \mathrm{b}}$, Victor Gustavo Balera Brito ${ }^{\mathrm{a}}$, Caril Constante Ferreira do Amaral ${ }^{\mathrm{a}, \mathrm{b}}$, \\ Antonio Hernandes Chaves-Neto a , Ana Paula Campanelli ${ }^{\mathrm{c}}$, Sandra Helena Penha Oliveira ${ }^{\mathrm{a}, \mathrm{b}, *}$ \\ a School of Dentistry of Araçatuba, UNESP - Univ Estadual Paulista, Campus Araçatuba, Department of Basic Sciences, São Paulo, Brazil

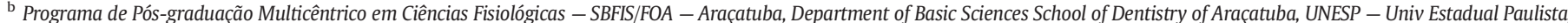 \\ Araçatuba, Department of Basic Sciences, São Paulo, Brazil \\ ' School of Dentistry of Bauru, USP - Universidade de São Paulo, Campus Bauru, Department of Biological Science, São Paulo, Brazil
}

\section{A R T I C L E I N F O}

\section{Article history:}

Received 2 September 2015

Received in revised form 23 December 2015

Accepted 9 January 2016

Available online 13 January 2016

\section{Keywords:}

Mesenchymal stem cells

Osteogenic differentiation

Spontaneously hypertensive rats (SHR)

Bone markers expression

Femur

\begin{abstract}
A B S T R A C T
Aims: Spontaneously hypertensive rats (SHR) and normotensive rats (W) has significant changes in bone metabolism. The purpose of this study was to investigate whether, the genetic predisposition, is sufficient to induce changes in the osteoblast differentiation and osteogenic markers in the BMSCs or in the femoral bone. For this we use young SHR rats without hypertension, but, with genetic predisposition in compared with young W. Main methods: BMSCs were cultured in a proliferation medium (MEM) or osteogenic medium. Osteogenic differentiation was analyzed by proliferation, total protein, alkaline phosphatase, mineralization, and the mRNA expression of RUNX-2, $\beta$-cathenin, osterix, bone morphogenetic protein-2 (BMP-2), osteocalcin (OCN), bone sialoprotein (BSP), collagen type I ( $\mathrm{Col} \mathrm{I}$ ), and osteopontin (OPN).

Key findings: Osteoblast differentiation in SHR BMSCs (SHRC) had an increased proliferation compared with W BMSCS (WC). After osteogenic induction, there was greater reduction in proliferation in SHR (SHROM) than in $\mathrm{W}$, in the same condition (WOM). On day 7, although no significant difference in the ALP activity was observed between SHROM and WOM, poor mineralization and osteoblast differentiation was noted in SHROM. The Osterix and $\beta$-catenin are involved in the reduced osteoblast differentiation in SHROM. The decreased expression of osteoblast-associated proteins such as OCN, BSP, COL I and OPN revealed poor quality of extracellular matrix (ECM) in SHROM. In the femoral bone, the immunostaining of COL1, BALP, OPN and OCN in SHR was decreased compared with the W. TRAP-positive immunoreactions were observed in major extension in the SHR femur. Significance: This study is the first to compare osteoblast differentiation in vitro and femoral bone from SHR and W rats. Our results demonstrated that young SHR (4 weeks old), without hypertension, but with genetic predisposition, had alterations in osteoblast differentiation of BMSCs and in the femoral bone when compared with their progenitor strain, $\mathrm{W}$.
\end{abstract}

(c) 2016 Elsevier Inc. All rights reserved.

\section{Introduction}

Bone marrow mesenchymal stem cells (BMSCs) are multipotent stem cells that have the ability to differentiation into osteoblasts and other cellular types, such as chondrocytes and adipocytes [1,2]. During in vitro osteogenesis, BMSCs form a multilayer and synthesize extracellular matrix with a bone tissue-like organization [3,4]. This process can be divided into three periods: (i) proliferation, (ii) extracellular matrix maturation and (iii) mineralization [5]. In the active proliferation stage, cells synthesize growth factors, collagen and transcription factors activation. After this stage, there is protein production related to

\footnotetext{
* Corresponding author at: Dept. of Basic Sciences, School of Dentistry of Araçatuba, Univ. Estadual Paulista-UNESP, Rodovia Marechal Rondon, Km 527, Bloco 31-CEP, 16018-805 Araçatuba, SP, Brazil.

E-mail address: shpoliv@foa.unesp.br (S.H.P. Oliveira).
}

extracellular matrix maturation and later mineralization, such as osteocalcin (OCN), bone sialoprotein (BSP), and alkaline phosphatase (ALP). The cessation of growth and extracellular matrix accumulation are maturation signals of the osteoblast phenotype [6]. All these proteins are expressed at maximal levels in the subsequent stage of differentiation at the onset of mineralization, considered a functional in vitro endpoint reflecting advanced cell differentiation [7].

Spontaneously hypertensive rats (SHR) are a model of human essential hypertension developed by Okamoto and Aoki [8]. This strain was obtained from Wistar rats (W) with a blood pressure above the average. Cardiac hypertrophy, diastolic dysfunction [9], metabolic acidosis [10], constitutive cytokine and chemokine production [11] and significant changes in bone metabolism, such as increased bone turnover and reduction in cortical and trabecular bone mass [12-15] are characteristics of this animals. This strain develops hypertension after 6 weeks of age and is therefore, a model of hypertension associated with bone 
problems. The hypertensive genotypic influence on the hyperproliferation activity on the smooth muscle cells (SMC) of young, fourweek-old SHR has been demonstrated in [16]. The purpose of this study was to investigate whether, the genetic predisposition, is sufficient to induce changes in the osteoblast differentiation and osteogenic markers in the BMSCs or in the femoral bone. For this we use young SHR rats (4 weeks old) without hypertension, but, with genetic predisposition in compared with young $\mathrm{W}$. The results demonstrated that there are differences in several parameters: A) SHR BMSCs have increased proliferative rate; B) Mineralization was lower in the SHROM (osteogenic medium) in comparison with the WOM; C) Reduced osteoblast differentiation was observed in SHROM and D) TRAP-positive immunostaining was higher in SHR than in $\mathrm{W}$ in the femur.

\section{Materials and methods}

\subsection{Animals and ethical aspects}

The Experimental Procedures were reviewed and approved by the Institutional Animal Welfare Committee at the School of Dentistry of Araçatuba (UNESP - Univ. Estadual Paulista, São Paulo, Brazil - Process No. 00716-2012). A total of 12 male W and 12 male SHR, 4 weeks old, weighing between 100 and $160 \mathrm{~g}$, were employed. W rats were used with control, because SHR was obtained from this strain [8]. The age of animals was selected to study the genetic predisposition. Tests with adult animals are part of another study from the laboratory to be published soon. These animals were kept in cages under constant and controlled room temperature $\left(22 \pm 2{ }^{\circ} \mathrm{C}\right)$ and humidity $(55 \% \pm 3 \%)$ in a 12 -hour light-dark cycle, and received water and standard food ad libitum. In order to obtain pool of Bone Marrow Mesenchymal Stem Cells (BMSCs) used in this study, the rats were euthanized with an overdose of halothane (Tanohalo; Cristália, Campinas, SP, Brazil).

\subsection{Isolation and culture of bone marrow mesenchymal stem cells}

Bone marrow was obtained from both femora, and flushed out using $20 \mathrm{~mL}$ of minimal essential medium (MEM) containing $10 \%$ fetal bovine serum (FBS), $2 \mathrm{mM}$-glutamine, and $1 \%$ antibiotics ( $100 \mathrm{U} / \mathrm{mL}$ penicillin $\mathrm{G}, 100 \mu \mathrm{g} / \mathrm{mL}$ streptomycin and $0.3 \mu \mathrm{g} / \mathrm{mL}$ amphotericin B (Sigma).The cell suspension was filtered through a $70-\mu \mathrm{m}$ size cell strainer and through 22 and 26-gauge needles. After rapid centrifugation, the cell pellet was suspended in culture medium and then seeded in $75 \mathrm{~cm}^{2}$ tissue culture flasks (Santa Cruz Biotechnology). The cells were grown in a humidified atmosphere containing $5 \% \mathrm{CO}_{2}$ at $37^{\circ} \mathrm{C}$. After 10 days, once confluent, the cells were harvested using trypsin-EDTA solution (Sigma). Nucleated cells were counted and then seeded in a subculture at $4.0 \times 10^{5}$ cells/well in 24-well tissue culture plates (Santa Cruz Biotechnology). Again, under subconfluence (80\%), the cells were cultured in a proliferation medium (MEM) or osteogenic medium (MEM supplemented with $10 \mathrm{nM} \beta$-glycerophosphate, $50 \mu \mathrm{g} / \mathrm{mL}$ ascorbic acid and $10 \mathrm{nM}$ dexamethasone). Thus, the cells were divided into 4 groups: 1) WC (BMSCs of Wistar cultivated in proliferation medium, without osteogenic induction); 2) WOM (BMSCs of Wistar cultivated in osteogenic medium, with osteogenic induction); 3) SHRC (BMSCs of SHR cultivated in proliferation medium, without osteogenic induction) and 4) SHROM (BMSCs of SHR cultivated in osteogenic medium, with osteogenic induction. These experimental conditions were used for all the experiments.

\subsection{Flow cytometry}

After obtaining a pool of bone marrow cells of each of the two strains, the cells were cultivated with proliferative medium until the $2^{\text {nd }}$ passage in $75 \mathrm{~cm}^{2}$ tissue culture flasks (Santa Cruz Biotechnology). Therefore, the cells were harvested after detachment with $0.25 \%$ trypsin (Gibco) and were fixed with $4 \%$ paraformaldehyde. There was approximately $1 \times 10^{6}$ cells/tube. Next, the cells were incubated for $30 \mathrm{~min}$. at $4{ }^{\circ} \mathrm{C}$ with $10 \mathrm{mg} / \mathrm{mL}$ of rat anti-mouse monoclonal antibody (CD90:RPE; CD29:RPE; CD54:FITC; CD45:FITC; or CD31:FITC AbDSerotec, Kidlington, Oxford, OX5 1 GE, UK). After this, the cells were centrifuged, the supernatant medium was removed and $200 \mu \mathrm{L}$ of uncompleted medium was added. Data were acquired with the Attune ${ }^{\mathrm{TM}}$ acoustic focusing cytometer system (Applied Biosystems ${ }^{\circledR}$, Foster City, CA, USA). Negative control IgG was used to exclude the auto fluorescence.

\subsection{Markers of osteogenic differentiation}

\subsubsection{MTT reduction assay}

The culture cell proliferation and viability of BMSCs were measured at time intervals of 0, 7 and 14 days using the classical MTT method [17].

\subsubsection{Total protein content}

The total amount of protein after time intervals of 0,7 and 14 days was quantified using the Lowry [18] method.

\subsubsection{Alkaline phosphatase activity}

The Alkaline Phosphatase (ALP) activity was assessed as the release of Thymolphthalein from Thymolphthalein monophosphate using a commercial kit (Labtest Diagnostica SA, Ribeirão Preto, SP, Brazil), a modification of the Roy method [19]. Values were normalized against protein concentration [18].

\subsubsection{Mineralization assay}

Mineralization in osteoblast cultures was determined by Alizarin Red S staining (Sigma) after 10 and 14 days in culture. Staining was quantified by adaption of the method described by Gregory [20].

\subsection{Real-time $q P C R$}

Total RNA were isolated using TRIzol reagent (Invitrogen, Life Technologies, Grand Island, NY, USA) according to the manufacturer's instructions. The total RNA from each sample was treated with DNAse I and reverse transcribed to complementary DNA (cDNA) using SuperScript ${ }^{\mathrm{TM}}$ II Reverse Transcriptase (Invitrogen) according to the manufacturer's protocol.

The relative mRNA levels were evaluated by quantitative RT-PCR using StepOne ${ }^{\mathrm{TM}}$ Real-Time PCR System (Applied Biosystems, Life Technologies, United Kingdom) and TaqMan ${ }^{\circledR}$ (Applied Biosystems, Life Technologies, USA). To use this reaction, inventoried assays labeled with FAM fluorophore provided by the same company: Osteocalcin (Bglap - Rn00566386_g1), osteopontin (SPP1 - Rn00563571_m1), bone sialoprotein (Ibsp - Rn00561414_m1), type 1 collagen (COL1A1 - Rn01463848_m1); Runx2 (Runx2 - Rn01512298_ml), osterix (osterix Rn02769744_s1 Sp7), $\beta$-catenin (CTNNB1 Rn00584431_g1); were used in parallel with fluorophore VIC, provided by the same company: $\beta$-actin (ACTB-Rn00667869_m1).

\subsection{Immunohistochemistry}

The femurs were removed and fixed in $4 \%$ neutral formalin solution for $48 \mathrm{~h}$, then demineralized by immersion for $45 \mathrm{~d}$ in a solution containing $10 \%$ ethylenediaminetetracetic acid. After this procedure, the femurs were dehydrated and embedded in paraffin. Semiserial longitudinal 3- $\mu \mathrm{m}$ thick sections were obtained and stained to determine the production of collagen type 1 (Col1), bone alkaline phosphatase (BALP), osteopontina (OPN), osteocalcina (OCN) and tartrate-resistant acid phosphatase (TRAP). Staining with hematoxylin and eosin was performed, and then analyzed by light microscopy to establish bone characteristics. For immunohistochemistry (IHC), the sections were deparaffinized and rehydrated in phosphate buffered saline (PBS). A primary antibody, diluted 1:250 in PBS and 

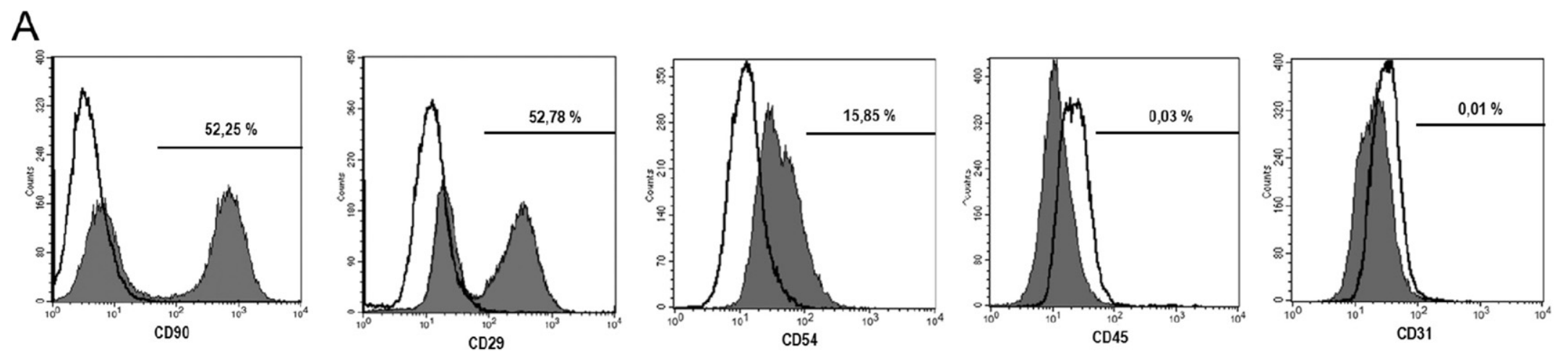

B
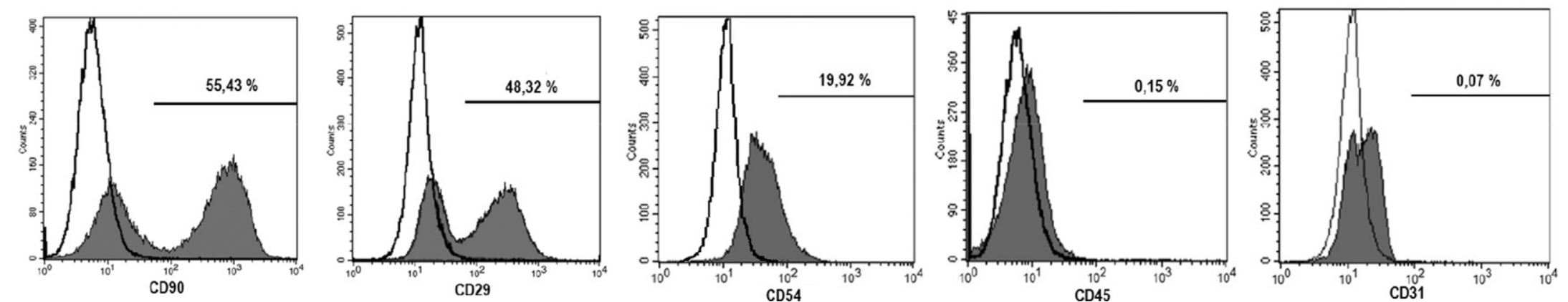

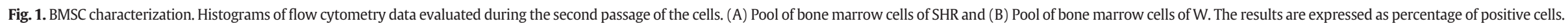



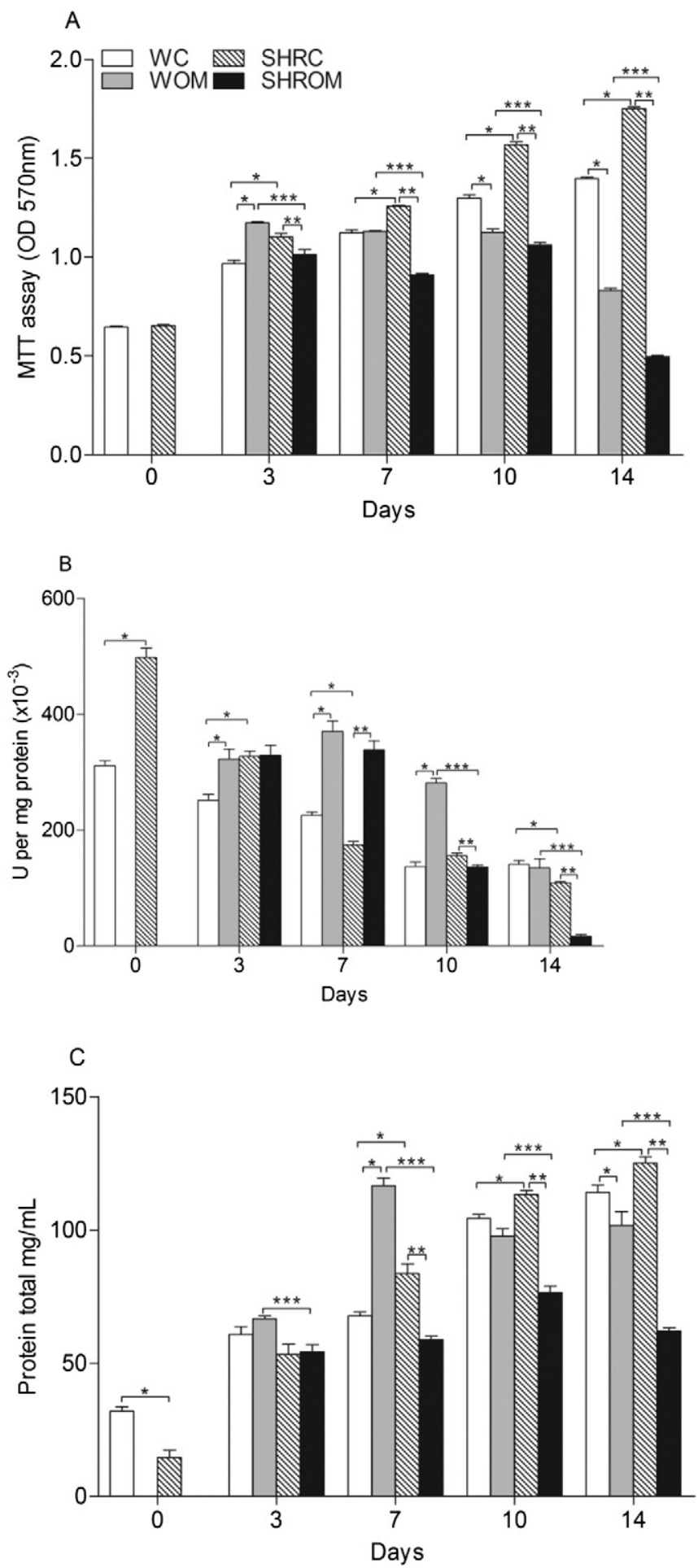

Fig. 2. Cell proliferation (A), alkaline phosphatase activity (B) and total protein content (C). These parameters were analyzed as a function of time (days) during the osteogenic differentiation process. Proliferation assay was expressed by OD (optical density). Alkaline phosphatase activity was expressed by amount of enzymatic activity per milligram of protein, the enzymatic activity unit (U) is the amount of ALP that catalyzes the transformation of $1 \mu \mathrm{mol}$ of substrate per minute at $37^{\circ} \mathrm{C}$ and $\mathrm{pH} 10.15$. The total protein content was expressed in $\mathrm{mg} / \mathrm{mL}$. The results are expressed as mean $\pm \mathrm{SEM}$. Groups were defined as: (WC) W BMSCs cultivated in proliferation medium; (WOM) W BMSCs in osteogenic medium; (SHRC) SHR BMSCs in proliferation medium; (SHROM) SHR BMSCs in osteogenic medium. Values ${ }^{*} \mathrm{p}<0.05$ versus WC: ${ }^{* *} \mathrm{p}<0.05$ versus SHRC; ${ }^{* * *} \mathrm{p}<0.05$ versus WOM. Triplicate samples were analyzed. Statistical analysis was performed using the two-way ANOVA test, followed by Bonferroni test.
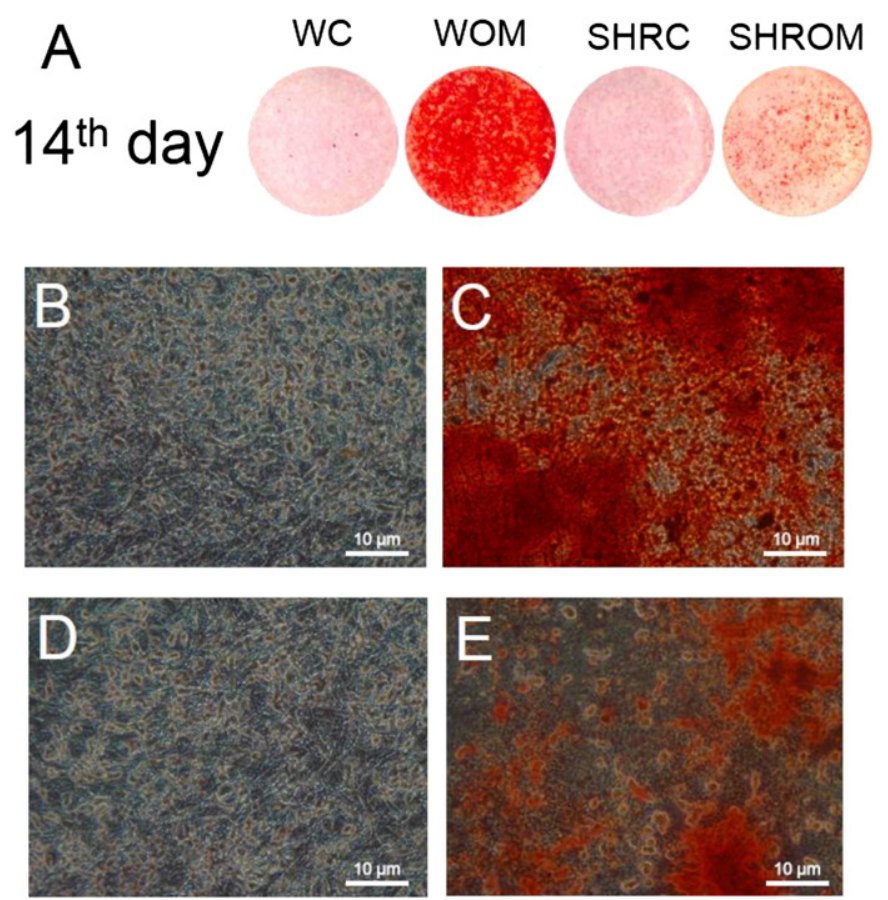

$\mathrm{F}$

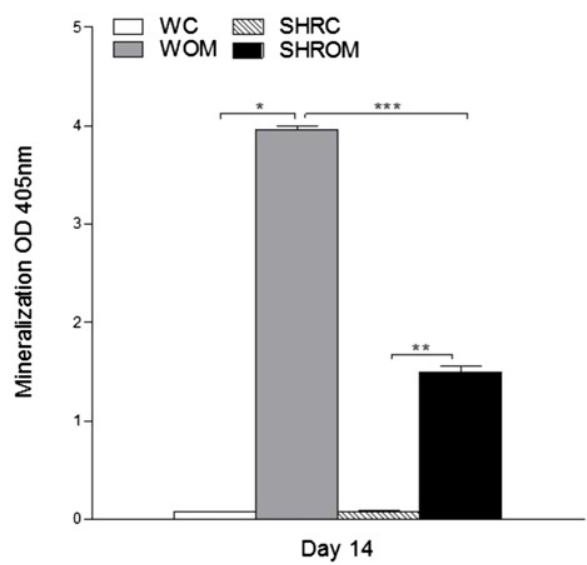

Fig. 3. Mineralization. Alizarin red staining analyses were performed after 14 days in culture. (A) 24-well cell culture plate, image captured by scanner; (B) WC group; (C) WOM group; (D) SHRC group; (E) SHROM group; (B-E) images captured by inverted microscope, $200 \times$ magnification. (F) Mineralization quantification, performed with colorimetric assays by extracting the alizarin red stain. The results are expressed as mean \pm SEM. Groups were defined as: (WC) W BMSCs cultivated in proliferation medium; (WOM) W BMSCs in osteogenic medium; (SHRC) SHR BMSCs in proliferation medium; (SHROM) SHR BMSCs in osteogenic medium. Values ${ }^{*} \mathrm{p}<0.05$ versus WC; ${ }^{* *} \mathrm{p}<0.05$ versus SHRC; ${ }^{* * *} \mathrm{p}<0.05$ versus WOM. Triplicate samples were analyzed. Statistical analysis was performed using the two-way ANOVA test, followed by Bonferroni test.

$0.1 \%$ bovine serum albumin (BSA), was performed at $4{ }^{\circ} \mathrm{C}$ for $16 \mathrm{~h}$. Then, it was incubated with a second antibody, diluted in PBS and BSA $0.1 \%$, for $60 \mathrm{~min}$ at room temperature. The avidin-biotin complex (Dako Corp., Carpinteria, CA, USA) was used to amplify the signal of the reaction developed with diaminobenzidine (Dako Corp.) as the chromogen. The procedure was completed by nuclear counterstaining using Harry's hematoxylin. All samples were accompanied by a negative control. Selected images were digitalized at high and low magnifications using a light microscope (Stemi 2000-C; Carl Zeiss do Brasil Ltda.) coupled with a digital camera (Axio Cam MRc5; Carl Zeiss do Brasil Ltda., Rio de Janeiro, RJ, Brazil). The background originating from digitalization, color balance, brightness, and contrast was corrected. 


\subsection{Western blot}

For Western blot analysis, cellular lysates were prepared and Western blot analysis was performed as previously described [21]. Cell extracts ( $75 \mu \mathrm{g}$ of protein) were separated by SDS-PAGE on $10 \%$ polyacrylamide gels and transferred to PVDF membranes. The appropriate primary antibody (ERK1/2, p-ERK1/2Thr202/Tyr204, SAPK/JNK, pSAPK/JNKThr183/Tyr185, p38, p-p38Thr180/Tyr182 e $\beta$-actina) (Santa Cruz, SC, CA, USA) was used at a 1:1000 dilution; and anti-mouse or anti-goat horseradish peroxidase-conjugated secondary antibodies diluted to 1:2000. Immunoreactive bands were detected with enhanced chemiluminescence. At least three Western blots were performed for each protein and the bands were quantified by densitometric analysis using the software program Image Analysis Scion (Scion Corp, Frederick, MD, EUA). The protein levels were expressed relative to $\beta$-actin.

\subsection{Statistical analysis}

All data were displayed as means \pm standard error of mean (SEM), analyzed by two-way ANOVA followed by Bonferroni post hoc test. The statistical software program GRAPHPAD PRISM 5.0 (GraphPad Software Inc., San Diego, CA, USA) was used. Values of $p<0.05$ were considered significant.

\section{Results}

\subsection{Positive expression of CD90, CD29 and CD54 characterized the} phenotype of bone marrow mesenchymal stem cells

Flow cytometry analysis was performed to characterize the phenotype of bone marrow cells obtained from $\mathrm{W}$ and SHR femurs. The results were positive for CD90, CD29 and CD54, specific surface markers of BMSCs, in both strains. In addition, the data were negative for CD45, hematopoietic surface marker, and for CD31, epithelial surface marker, in both strains (Fig. 1).

\subsection{Reduced proliferative rate was more pronounced in SHR BMSCS cultivated in osteogenic medium}

A time-dependent increase in proliferation rate was observed in both W and SHR C groups, indicating the ability to maintain proliferation during long-term culture. Nevertheless, the SHRC group showed a higher proliferation rate than the WC group. However, a reduced proliferation rate was observed in the OM groups compared with $\mathrm{C}$ groups, mainly at 14 day. When only the OM groups were compared, a poor proliferation rate was observed in the SHROM group when compared with the WOM group (Fig. 2A).

\subsection{The ALP activity peaked on day 7 in WOM and SHROM}

ALP, an early osteoblast marker, is thought to increase and then decrease when mineralization was well in progress. In Fig. 2B, ALP activity reflects the ALP concentration normalized by total protein content. In WC and SHRC, the ALP activity decreased in a time-dependent manner. The enzyme activity peak for WOM occurred at the 7th day and for SHROM a slight increase was observed at day 7, decreasing at 10 to 14 days.
3.4. The total protein content was reduced in SHROM in comparison with WOM

The determination of Total Protein Contents is important in order to evaluate the amount of organic matrix. The pattern of response of total protein (Fig. 2C) in both $\mathrm{C}$ groups was similar to the pattern of response of the proliferation assay (Fig. 2A), with a continuous increase during the time of culture. In the SHROM group the total protein levels were lower in comparison with those of WOM in all periods analyzed.

\subsection{Mineralization was reduced in SHROM}

Alizarin red staining was used to evaluate the presence of mineralization nodules. Mineralized nodules were observed only in cells cultured in osteogenic medium. Alizarin Red staining resulted in marked differences between $\mathrm{W}$ and SHR cultures at 14 days confirming osteoblast differentiation. However, in the SHROM group, mineralization was reduced in comparison with the WOM group (Fig. 3).

\subsection{Transcription factors osterix and $\beta$-catenin were reduced in the osteoblastic differentiation in SHROM}

To investigate the transcription factors involved in the osteoblast differentiation gene expression, real-time PCR analysis was performed. Runx2 expression (Fig. 4A) was similar between WOM and SHROM. However, the Osterix expression (Fig. 4B) was increased in WOM compared with SHROM and WC, but in SHROM Osterix expression was decreased when compared with SHRC. Moreover, the $\beta$-catenin expression (Fig. 4C) was increased in the WOM group in comparison with the SHROM group, but the expression in the SHROM group was reduced when compared with the SHRC group.

\subsection{Extracellular matrix proteins expression were was lower in SHROM}

Osteoblast-associated expression of ECM proteins, such as OCN (Fig. 4D), BSP (Fig. 4E), COL I (Fig. 4F) and OPN (Fig. 4G) was increased in WOM compared with SHR OM. The expression of these proteins was enhanced in OM groups in comparison with the $\mathrm{C}$ groups. The Col I expression was also increased in WC and SHRC group where we had only femur BMSCs without osteogenic medium (Fig. 4F).

\subsection{TRAP-positive cells immunostaining is more pronounced in SHR femur}

To confirm whether SHR shows poor extracellular matrix with less immunostaining to bone markers and more TRAP immunostainingpositive cells, we analyzed the trabecular zone localized in the metaepiphyseal portion of the femoral bone by the immunohistochemistry of some bone-relevant marker genes, including Col1, BALP, OPN, OCN and TRAP. In H\&E histology showed that there are more space in the trabecular bone in SHR compared with the $\mathrm{W}$ animals (Fig. 5A, B). Col I-immunoreaction cells were detected in the bone-marrow, in preosteoblast and osteoblast, on both groups, however, the major immunostaining can be observed in W compared with the SHR (Fig. 5C, D). BALP-immunoreaction cells were observed in osteoblasts near bone matrix, in higher intensity in $\mathrm{W}$ (Fig. 5E, F). OPN-immunostaining became more intense in bone-marrow, in pre-osteoblast and osteoblast from W compared with the SHR, although in these animals the

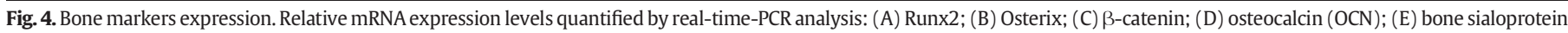

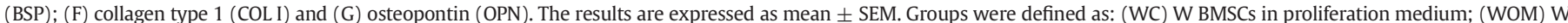

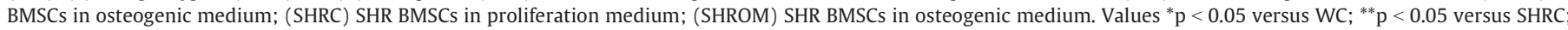

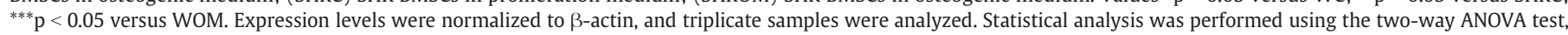
followed by Bonferroni test.
} 

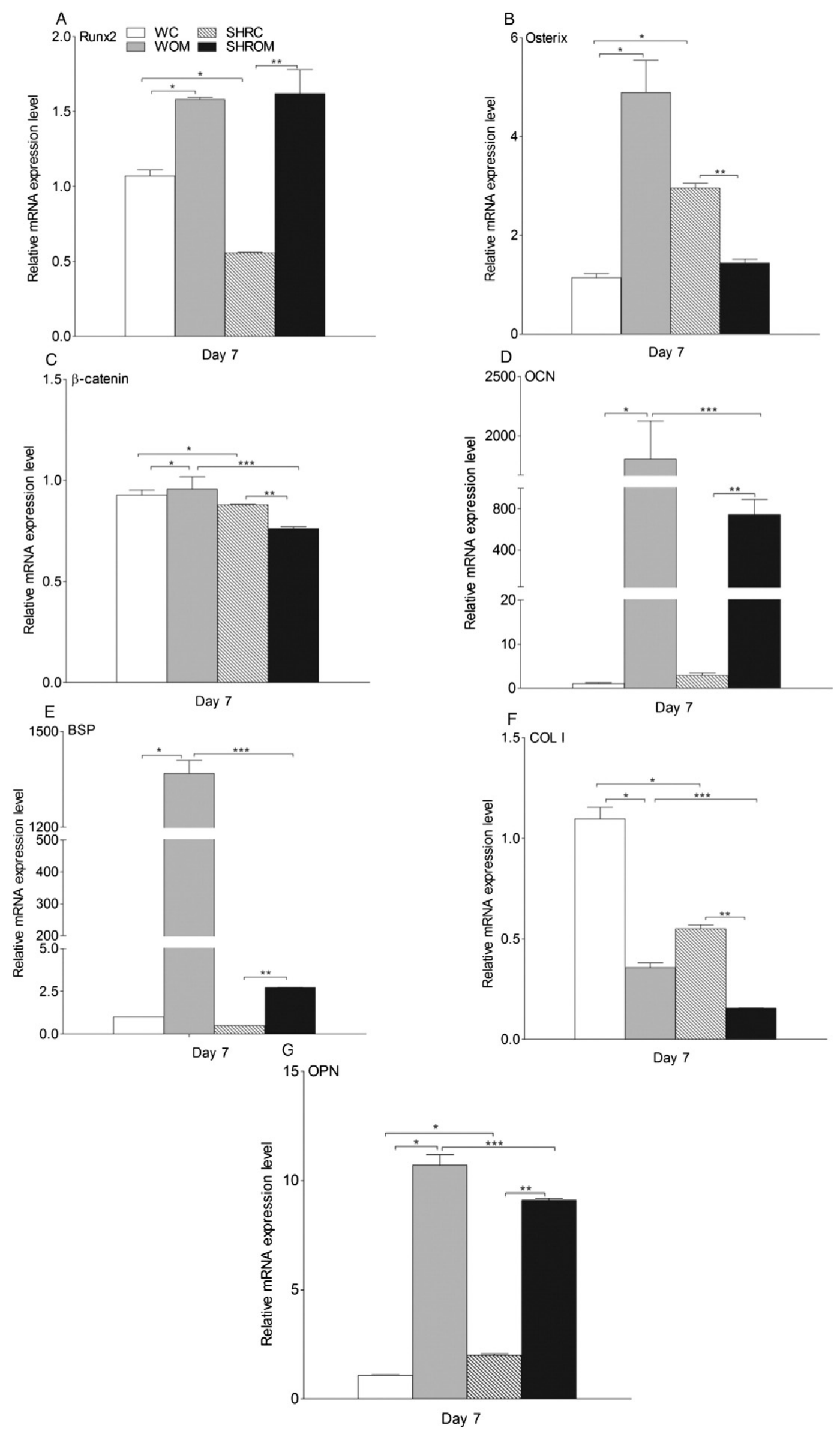

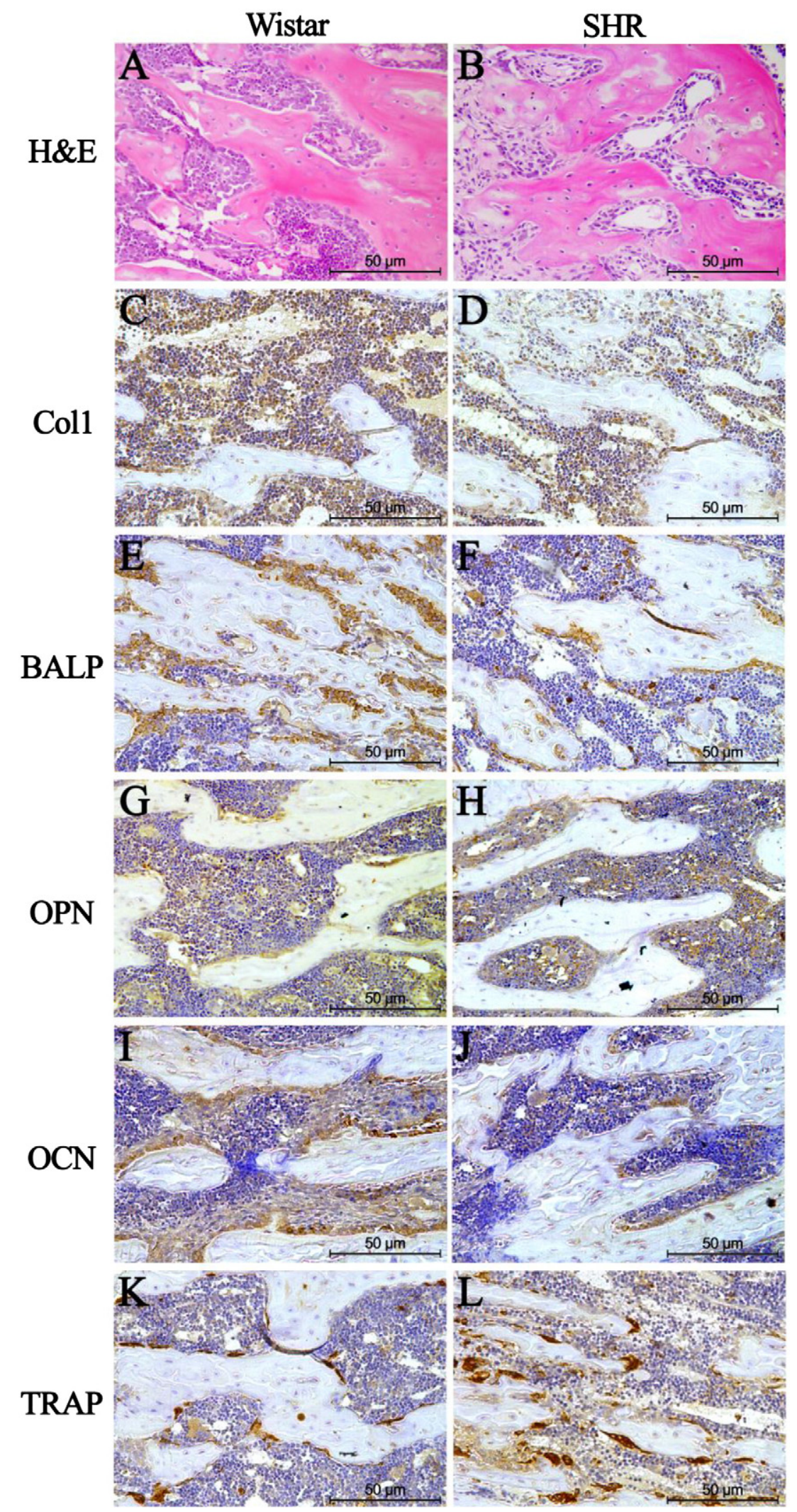

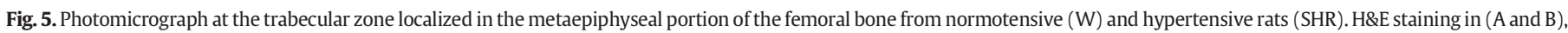

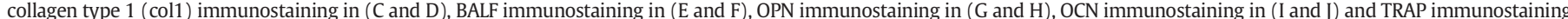
(K and L). Original magnification: $100 \times$.

pattern of immunoreaction was significant (Fig. 5G, H). In W animals, $\mathrm{OCN}$-immunoreaction is more pronounced in osteoblast, compared those observed in the SHR osteoblast, in minor amount near marrow space (Fig. 5I, J). In the TRAP-positive immunoreaction is visible pronounced increase in the number of osteoclast in SHR compared with W (Fig 5K, L). 


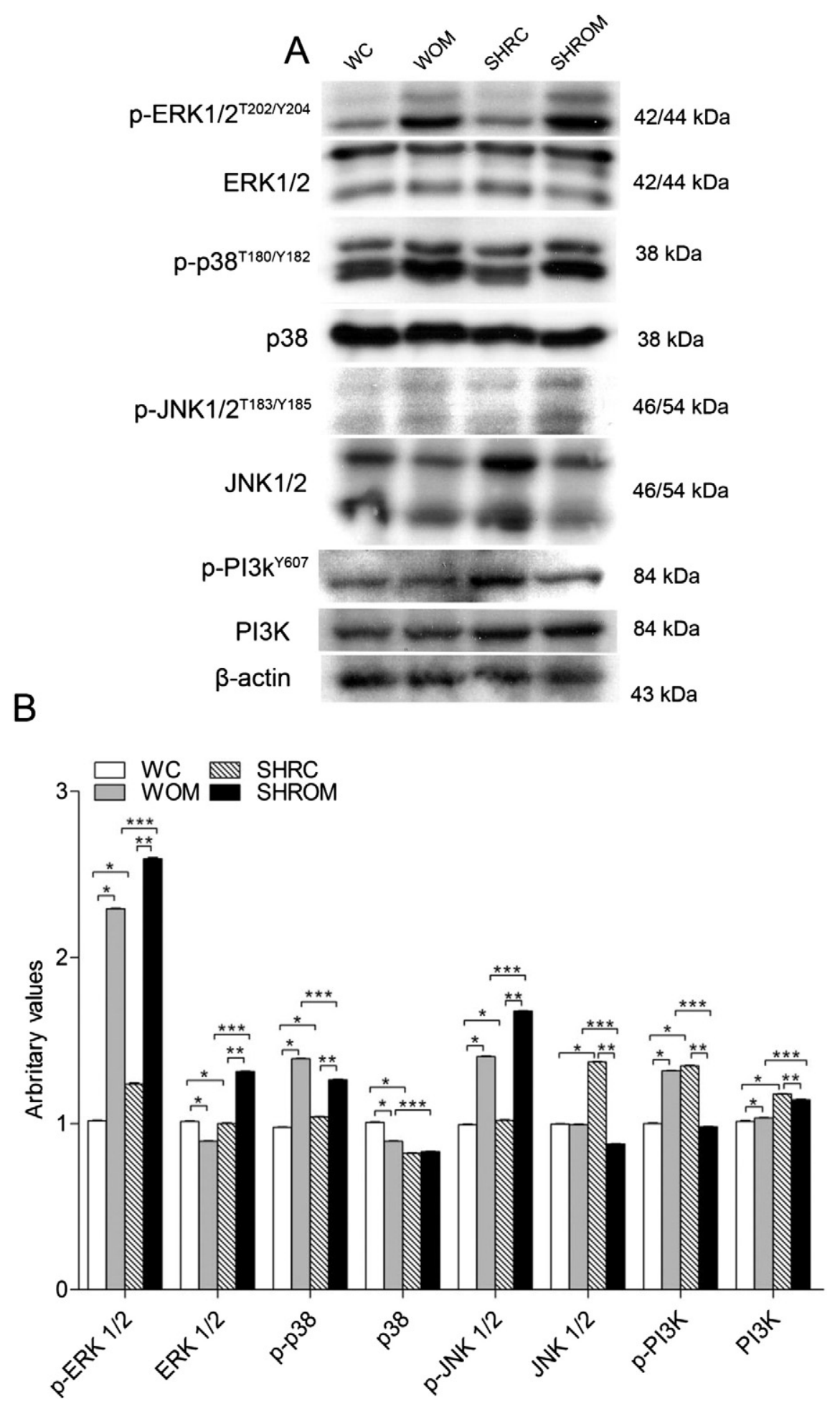

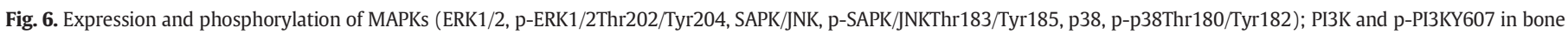

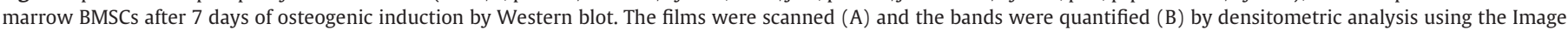

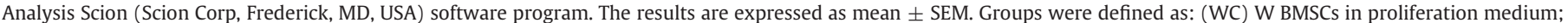

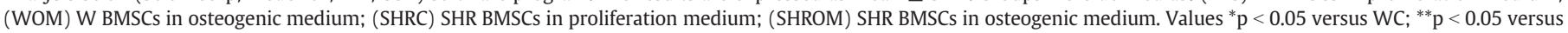
SHRC. Triplicate samples were analyzed. Statistical analysis was performed using the two-way ANOVA test, followed by Bonferroni test.

3.9. Activation of MAPKs phosphorylated in OM groups during osteogenic differentiation

To investigate the intracellular signaling pathways involved in the osteogenic differentiation of BMSCs, we analyzed the MAPKs and PI3K signaling pathways via Western blotting (Fig. 6). An increase in the phosphorylation of ERK 1/2, JNK $1 / 2$ and p38 was observed in the OM groups when compared with the respective controls. The activation of ERK 1/2 was greater in WOM and SHROM groups in comparison with the other MAPKs. Furthermore, a pronounced increase in ERK 1/2 and 
JNK $1 / 2$ phosphorylation was observed in the SHROM group in the period analyzed. However, there was greater $\mathrm{p} 38$ phosphorylation activity in the WOM group. With regard to PI3K activity, a higher level of phosphorylation was observed in the WOM group compared with the WC group, and in the SHRC group when compared with the SHROM group.

\section{Discussion}

These finding are the first to compare osteoblast differentiation in vitro and femoral bone from SHR and W rats. Our results demonstrated that young SHR (4 weeks old), without hypertension, but with genetic predisposition, had alterations in osteoblast differentiation of BMSCs and in the femoral bone when compared with their progenitor strain, $\mathrm{W}$.

First of all, to confirm the purity of BMSCs, characterization was performed showing positivity for CD29, CD90 and CD54 markers, indicating the presence of BMSCs in the pool of bone marrow cells [22,23]. However, the percentage of BMSCs suggests subculture heterogeneity in the study period. This may be due to low passage cells (passage 2 ) used for characterization. Studies have reported that the greater is the passage of the cells, the best cell purity will be obtained [24,25]. An important point was the negativity for CD31 and CD45, markers for epithelial and hematopoietic cells [26], respectively, once again showing that the pool of cells present in bone marrow is mesenchymal stem cells.

The SHRC and WC are groups with undifferentiated BMSCs. They sustained continuous growth and showed proliferation ability, which was maintained during long-term culture. Nevertheless, SHRC showed higher proliferation rates than WC, showing evidence of hyperproliferation of SHR from BMSCs. The hypertensive genotype influence, on the hyperproliferation, was also observed in SMC and fibroblast from thoracic aorta of 4-week-old SHRs, where changes in growth patterns of aortic cells isolated from young prehypertensive SHR seem to be restricted to SMC and fibroblasts [16]. In our data, in the first three days of the differentiation, in OM groups were observed grown during the onset of osteogenesis, in proliferative period [5]. After this period (days 7 and 10), a continuous proliferation rate was noted in WOM and SHROM, demonstrating that the BMSCs stopped the proliferation process, to begin differentiating into osteoblasts [27], decreasing after day 14. In SHR BMSCs submitted to osteogenic induction (SHROM) the proliferation rate decreased more than it did in $\mathrm{W}$, in the same condition, compared with the respective control, demonstrating greater sensitivity to osteogenic induction in SHR BMSCs. In previous experiments with a lower density, the osteogenic induction caused the death of SHR BMSCs, therefore we had to increase the density of cells to perform this study. This death occurred because the SHR BMSCs is more sensitive to osteogenic medium than the W BMSCs.

The cessation of growth and accumulation of extracellular matrix (ECM) are maturation signals of the osteoblast phenotype reflected by up regulation of alkaline phosphatase (ALP), an essential marker for matrix mineralization [27], characterizing the second stage of osteogenesis, in the extracellular matrix maturation period [5]. In our results, in OM groups, higher ALP activity was observed on day 7, and mineralization was noted in both cultures on day 10 (data not shown), confirming that the enhanced ALP levels were an early marker of mineralization. Nevertheless, there was no difference in ALP activity between WOM and SHROM on day 7, indicating that ALP activity was not responsible for the lower mineralization in SHROM. Another observation was lower total protein content in SHROM in comparison with WOM. This result suggests the poor quality of the extracellular matrix in SHROM. This can be confirmed with the expression of mRNA, since demonstrate osteogenic markers is decreased in SHROM. In studies using osteogenic cultures, mineralization is considered a functional in vitro endpoint reflecting advanced cell differentiation [7]. In the mineralization assay, the detection of mineralized nodules was lower in SHROM when compared with WOM in the same conditions. Taken together, we suggest that greater decrease in proliferation rate and lower total protein content after osteogenic induction in comparison with the respective control could be evidence of a lower level of differentiation. Consequently, the lower degree of mineralization in SHROM may be due to poor quality ECM and a lower level of differentiation, and not due to weak ALP activity.

Up to now, these finding indicate that, in comparison with W, SHR showed a lower level of osteoblast differentiation of BMSCs and consequently a lower degree of mineralization. After these initial results, we decided to evaluate the expression of osteogenic markers. First of all, the transcription factors Runx2, Osterix and $\beta$-catenin were analyzed. Runx2 is an important transcription factor necessary for the initiation of osteoblast differentiation and bone formation [28]. In our results, the relative quantification of Runx2 was higher in OM groups than in C groups, but no differences were observed between SHROM and WOM, suggesting that Runx2 is not the signal that causes reduced differentiation in SHROM. Osterix functions occur and are downstream from Runx2 during osteoblast differentiation, and several transcription factors regulate osteoblast differentiation by regulating Osterix [29], including $\beta$-catenin, a transcription cofactor dependent on the Wnt signaling pathway. In our results, although there was no difference in the Runx2 expression activation between SHROM and WOM, Osterix expression was decreased in SHROM, suggesting that this transcription factor could be responsible for the lower differentiation in SHROM, and it could be done via $\beta$-catenin [30], whereas the $\beta$-catenin expression was also lower in SHROM. The $\beta$-catenin also contributed directly to osteoblast differentiation [31,32], therefore this was another transcription cofactor shown to be lower in SHROM. Together, these results confirm the reduced osteoblast differentiation of SHR BMSCs.

With regard to the osteoblast-associated proteins, our study revealed differences between the ECM of SHROM and WOM. Among these proteins, we evaluated $\mathrm{OCN}$, which binds calcium and promotes bone matrix calcification [27]; BSP, a hydroxyapatite nucleator [33]; OPN, associated with cell attachment [34] and Col I. These proteins are the main EMC components that support proliferation, matrix formation and mineralization. Our data showed that all proteins were decreased in SHROM when compared with WOM, confirming the poor quality of ECM of SHR demonstrated by lowest total protein content and decreased expression of osteogenic markers mRNA. These results were confirmed using in situ study, where we observed that immunostaining to Col I, BALP, OPN, OCN in femur from $W$ was higher than in SHR. Furthermore, we found that Col I, in vitro, is overexpressed in the WC and SHRC group without osteogenic medium. I-Collagen protein is the most abundant extracellular protein in bone and is expressed during all stages of osteoblast development [35]. In our study, in situ, using femoral bone, the BMSCs from W and SHR are also able to produce a great amount of $\mathrm{Col}$ I, we can correlate this fact observing an increase of Col-I-immunostaining in the femoral bone from $\mathrm{W}$, mainly in the pre- and osteoblast, compared with SHR. It suggests that there are higher amount of osteogenic products may be important to improve the matrix mineralization in W than in the SHR (Fig. 5). Furthermore, the amount of TRAP-positive immunoreactions was higher in SHR than $\mathrm{W}$, showing the increase in the amount of osteoclast cells in the bone matrix instead osteoblast. TRAP is secreted in large quantities by osteoclasts, demonstrated by histochemical studies, so that it is considered a biochemical marker of osteoclast function [36]. These cells may contribute, at least in part, to the bone resorption process, since their adherence to bone is associated with an increased true osteoclast [37]. In conclusion, we suggest that SHROM had a lower level of differentiation and poor quality ECM resulting in lower mineralization.

After elucidation of the differences in ECM between WOM and SHROM groups in relation on constituents of bone matrix, we investigated whether the intracellular signaling pathways of MAPKs and PI3K could be involved in the process. The results showed the involvement of MAPKs, ERK 1/2, JNK $1 / 2$ and p38 in the differentiation of BMSCs in both strains, because there was increased phosphorylation 
in the OM groups. The ERK $1 / 2$ phosphorylation in the OM groups was shown to be pronounced in comparison with other MAPKs, in the study period (day 7 ). Studies have reported that in the early periods of osteogenic differentiation, ERK $1 / 2$ shows a marked increase in phosphorylation, indicating active participation in this period, as has been noted by other authors [38,39]. Moreover, corroborating our data, these authors reported that JNK $1 / 2$ and $\mathrm{p} 38$, which showed more discrete activity compared with ERK $1 / 2$ at the 7 th day, were more active later during osteogenic differentiation $[38,39]$. Although there was lower expression of matrix proteins and mineralization in SHRMO group, there were no remarkable correlations to MAPK signaling pathways, suggesting that these pathways are not responsible for such signaling responses. However, these pathways could be related to other cellular events such as cell proliferation [38]. Thus, further studies are necessary to elucidate other possible signaling pathways involved in the SHRMO response.

In summary, our findings indicate that SHR, without hypertension, but with genetic predisposition, showed decreased osteoblast differentiation of the BMSCs, poor quality of the ECM, lower mineralization and weak femoral bone. However, more studies are needed to show the mechanism involved in the bone metabolism of young SHR.

\section{Acknowledgments}

Thamine Landim de Barros was supported by a Master scholarship from Fundação de Amparo à Pesquisa do Estado de São Paulo (FAPESP) \# 2012/01924-9 and Grant Nos. 2011/06070-5 and 2011/19458-1.

\section{References}

[1] A.J. Friedenstein, et al., Heterotopic of bone marrow. Analysis of precursor cells for osteogenic and hematopoietic tissues, Transplantation 6 (2) (1968) 230-247.

[2] C. Maniatopoulos, J. Sodek, A.H. Melcher, Bone formation in vitro by stromal cells obtained from bone marrow of young adult rats, Cell Tissue Res. 254 (2) (1988) 317-330.

[3] J.E. Aubin, et al., Osteoblast and chondroblast differentiation, Bone 17 (2 Suppl.) (1995) 77S-83S.

[4] J.B. Lian, G.S. Stein, Development of the osteoblast phenotype: molecular mechanisms mediating osteoblast growth and differentiation, Iowa Orthop. J. 15 (1995) 118-140.

[5] T.A. Owen, et al., Progressive development of the rat osteoblast phenotype in vitro: reciprocal relationships in expression of genes associated with osteoblast proliferation and differentiation during formation of the bone extracellular matrix, J. Cell. Physiol. 143 (3) (1990) 420-430.

[6] C. Ratisoontorn, et al., In vitro differentiation profile of osteoblasts derived from patients with Saethre-Chotzen syndrome, Bone 36 (4) (2005) 627-634.

[7] C.D. Hoemann, H. El-Gabalawy, M.D. McKee, In vitro osteogenesis assays: influence of the primary cell source on alkaline phosphatase activity and mineralization, Pathol. Biol. (Paris) 57 (4) (2009) 318-323.

[8] K. Okamoto, K. Aoki, Development of a strain of spontaneously hypertensive rats, Jpn. Circ. J. 27 (1963) 282-293.

[9] S. Dupont, et al., The onset of left ventricular diastolic dysfunction in SHR rats is not related to hypertrophy or hypertension, Am. J. Physiol. Heart Circ. Physiol. 302 (7) (2012) H1524-H1532.

[10] K. Akira, et al., A metabonomic study of biochemical changes characteristic of genetically hypertensive rats based on (1)H NMR spectroscopic urinalysis, Hypertens. Res. 35 (4) (2012) 404-412.

11] C.F. Bonato, et al., Hypertension favors the inflammatory process in rats with experimentally induced periodontitis, J. Periodontal Res. 47 (6) (2012) 783-792.
[12] Y. Izawa, et al., Bone disorders in spontaneously hypertensive rat, Calcif. Tissue Int 37 (6) (1985) 605-607.

[13] M. Barbagallo, et al., Histological evidence of increased turnover in bone from spontaneously hypertensive rats, Cardioscience 2 (1) (1991) 15-17.

[14] T.M. Wang, et al., Evidence for reduced cancellous bone mass in the spontaneously hypertensive rat, Bone Miner. 20 (3) (1993) 251-264.

[15] G.L. Wright, D. DeMoss, Evidence for dramatically increased bone turnover in spontaneously hypertensive rats, Metabolism 49 (9) (2000) 1130-1133.

[16] T. Battle, J.F. Arnal, J.B. Michel, Hyperproliferation of aortic smooth muscle cells and fibroblasts from young SHR rats is not shared by endothelial cells, Clin. Exp. Pharmacol. Physiol. 21 (12) (1994) 981-989.

[17] D.C. Zancanela, et al., The effect of photosensitizer drugs and light stimulation on osteoblast growth, Photomed. Laser Surg. 29 (10) (2011) 699-705.

[18] O.H. Lowry, et al., Protein measurement with the Folin phenol reagent, J. Biol. Chem. 193 (1) (1951) 265-275

[19] A.V. Roy, Rapid method for determining alkaline phosphatase activity in serum with thymolphthalein monophosphate, Clin. Chem. 16 (5) (1970) 431-436.

[20] C.A. Gregory, et al., An Alizarin red-based assay of mineralization by adherent cells in culture: comparison with cetylpyridinium chloride extraction, Anal. Biochem. 329 (1) (2004) 77-84.

[21] A.H. Chaves Neto, et al., Profiling the changes in signaling pathways in ascorbic acid/ beta-glycerophosphate-induced osteoblastic differentiation, J. Cell. Biochem. 112 (1) (2011) 71-77.

[22] R.H. Lee, et al., Characterization and expression analysis of mesenchymal stem cells from human bone marrow and adipose tissue, Cell. Physiol. Biochem. 14 (4-6) (2004) 311-324.

[23] Y. Yu, et al., Mesenchymal stem cells over-expressing hepatocyte growth factor improve small-for-size liver grafts regeneration, Mol. Ther. 15 (7) (2007) 1382-1389.

[24] M.M. Fariha, et al., Human chorion-derived stem cells: changes in stem cell properties during serial passage, Cytotherapy 13 (5) (2011) 582-593.

[25] J.B. Mitchell, et al., Immunophenotype of human adipose-derived cells: temporal changes in stromal-associated and stem cell-associated markers, Stem Cells 24 (2) (2006) 376-385.

[26] M. Dezawa, et al., Specific induction of neuronal cells from bone marrow stromal cells and application for autologous transplantation, J. Clin. Invest. 113 (12) (2004) 1701-1710.

[27] J.B. Lian, et al., Osteocalcin gene promoter: unlocking the secrets for regulation of osteoblast growth and differentiation, J. Cell. Biochem. Suppl. 30-31 (1998) 62-72.

[28] P. Ducy, et al., Osf2/Cbfa1: a transcriptional activator of osteoblast differentiation, Cell 89 (5) (1997) 747-754.

[29] F. Long, Building strong bones: molecular regulation of the osteoblast lineage, Nat. Rev. Mol. Cell Biol. 13 (1) (2012) 27-38.

[30] X. Tu, et al., Noncanonical Wnt signaling through G protein-linked PKC delta activation promotes bone formation, Dev. Cell 12 (1) (2007) 113-127.

[31] Y. Chen, et al., Reciprocal interferences of TNF-alpha and Wnt1/beta-catenin signaling axes shift bone marrow-derived stem cells towards osteoblast lineage after ethanol exposure, Cell. Physiol. Biochem. 32 (3) (2013) 755-765.

[32] T.F. Day, et al., Wnt/beta-catenin signaling in mesenchymal progenitors controls osteoblast and chondrocyte differentiation during vertebrate skeletogenesis, Dev. Cell 8 (5) (2005) 739-750.

[33] K. Vincent, M.C. Durrant, A structural and functional model for human bone sialoprotein, J. Mol. Graph. Model. 39 (2013) 108-117.

[34] E. Donzelli, et al., Mesenchymal stem cells cultured on a collagen scaffold: In vitro osteogenic differentiation, Arch. Oral Biol. 52 (1) (2007) 64-73.

[35] T. Wurtz, et al., Collagen mRNA expression during tissue development: the temporospatial order coordinates bone morphogenesis with collagen fiber formation, Matrix Biol. 17 (5) (1998) 349-360.

[36] C. Minkin, Bone acid phosphatase: tartrate-resistant acid phosphatase as a marker of osteoclast function, Calcif. Tissue Int. 34 (3) (1982) 285-290.

[37] G. Vaes, Cellular biology and biochemical mechanism of bone resorption. A review of recent developments on the formation, activation, and mode of action of osteoclasts, Clin. Orthop. Relat. Res. 231 (1988) 239-271.

[38] R.K. Jaiswal, et al., Adult human mesenchymal stem cell differentiation to the osteogenic or adipogenic lineage is regulated by mitogen-activated protein kinase, J. Biol. Chem. 275 (13) (2000) 9645-9652.

[39] A. Suzuki, et al., Evidence for a role of p38 MAP kinase in expression of alkaline phosphatase during osteoblastic cell differentiation, Bone 30 (1) (2002) 91-98. 